\title{
Desafío en la enseñanza de español/L2 en comunidades quechua
}

\author{
Matilde Pérez Descalzo. Universidad de Granada \\ Juan Ramón Guijarro Ojeda. Universidad de Granada - ORCID 0000-0001-8453-2909
}

\author{
Recepción: 26/06/2018 | Aceptado: 17/04/2019 \\ Correspondencia a través de ORCID: Matilde Pérez-Descalzo
}

0000-0002-0807-5969

Citar: Pérez Descalzo, M y Guijarro Ojeda, JR (2019). Desafío en la enseñanza de español/L2 en comunidades quechua. ReiDoCrea - Monográfico sobre Perspectivas transnacionales en la enseñanza de lenguas, 8(3),191-201.

\begin{abstract}
Resumen: En la actualidad asistimos a un período en el que el uso de las TIC ha suscitado un gran cambio en la educación, convirtiéndose en uno de los ejes centrales en el proceso de enseñanzaaprendizaje. Sin embargo, nos encontramos con entornos educativos en los que resulta inviable el uso de nuevas tecnologías. En el contexto que nos ocupa -comunidades indígenas en Perú- la posibilidad de emplear herramientas tecnológicas es prácticamente inexistente. Tras la investigación llevada a cabo en dichas comunidades con el objetivo de favorecer la adquisición de la L2 mediante el análisis del entorno en el que se produce el proceso de enseñanza-aprendizaje y la metodología y los materiales didácticos empleados en la enseñanza de la lengua, en educación infantil y primaria, surge la necesidad de ofrecer un método adecuado que garantice la calidad educativa. Los resultados obtenidos nos llevan a concluir que la inadecuación de los recursos didácticos, entre otros, dificultan la adquisición de la L2. Por ello, se ha creado un manual -Derecho a comunicar- con el que se pretende: fomentar el desarrollo de destrezas; introducir conceptos específicos de diferentes disciplinas y potenciar los derechos del niño y la educación en valores a través de la enseñanza de la lengua.
\end{abstract}

Palabras clave: enseñanza de una segunda lengua; población indígena

\section{Challenges in the teaching of Spanish as a Second Language in Quechua communities}

\begin{abstract}
In recent years Information and Communication Technologies (ICTs) has brought important changes to education, rapidly becoming a central pillar in the teaching-learning process. The so-called ICTs are instruments with great didactic potential, offering a wide range of resources that encourage motivation and learning. There are, though, educational environments in which, for different reasons, the use of new technologies is unfeasible. In the context that concerns us - indigenous communities in the district of Huancarani in Peru - the possibility of using any technological tool is practically non-existent. Research into methodology and materials used in these non-ICT language 2 classrooms in infant and primary education, demonstrates the need for new teaching methods and the development of an environment that guarantees educational quality. Results show that inadequacy of teaching resources, among other things, hinders L2 acquisition. The objective of this project was to create a manual which seeks to facilitate language learning within this environment of limited technological resources, focusing on the needs of students living in a community in a challenged socio-economic situation. The didactic proposal - Right to Communicate - aims to promote the development of linguistic, cognitive and emotional skills, to use the L2 as a vehicle for civil and ethical education (including the rights of the child) and to encourage motivation in learning by using activities with a strong recreational component.
\end{abstract}

Keywords: Second language instruction; Indigenous people

\section{Introducción}

Las nuevas tecnologías se han ido convirtiendo en una herramienta didáctica fundamental que nos permiten mostrar contenidos de forma lúdica y visualmente atractiva para nuestros alumnos. Actualmente, los docentes tenemos a nuestra disposición una amplia gama de recursos -blogs, plataformas, podcast, redes sociales, etc.- que podemos emplear en el aula. $\mathrm{Y}$, a pesar de las dificultades que han 
ido apareciendo con la introducción de las TIC en el ámbito educativo, tales como: falta de recursos tecnológicos en los centros educativos; brecha digital entre alumnos y profesores; uso inapropiado de los recursos, etc., son indudables las ventajas que las nuevas tecnologías nos brindan.

A pesar de ello, en un momento en el que apenas se concibe la enseñanza sin el uso de las TIC, nos enfrentamos al desafío de enseñar de una forma que, a priori, para algunos docentes podría resultar anacrónica y obsoleta desde una perspectiva didáctica. Sin embargo, en contextos en los que el desarrollo tecnológico es prácticamente inexistente y la posibilidad de utilizar nuevas tecnologías resulta inviable, es necesario crear material específico que cubra las necesidades educativas de los alumnos mediante actividades que se adecuen a sus características y a las del entorno en el que se lleva a cabo la enseñanza. Por otra parte, es necesario poner de manifiesto que el hecho de que no podamos emplear ciertas herramientas, no excluye una enseñanza de calidad.

En el caso que nos ocupa- las comunidades rurales quechua hablantes del distrito de Huancarani (Paucartambo, Cuzco) en Perú- existen numerosos factores socioeconómicos que no permiten el uso de tecnología. Asimismo, desde un punto de vista educativo, como veremos al analizar el material didáctico, aprender una L2 a partir de conceptos abstractos ajenos a su conocimiento, entre otros, no favorece el aprendizaje. Por lo tanto, debemos adecuar el material didáctico al contexto y al alumno e incluir nuevos conceptos, bajo una perspectiva interdisciplinar, e introducir recursos didácticos desconocidos para el grupo -nuevas tecnologías- de manera graduada. Si bien es cierto que en las comunidades objeto no se puede hacer uso de la tecnología, resulta indispensable revisar la metodología que se emplea en la enseñanza de la L2, ya que se pueden transmitir conocimientos con materiales de calidad en soporte papel, sin recurrir a formatos digitales.

La propuesta didáctica que se ofrece pretende dar respuesta a las necesidades educativas -principalmente lingüísticas- de los niños del distrito, obtenidas a través de la investigación etnográfica realizada durante un periodo de voluntariado de ocho meses en la zona, con el objetivo de favorecer el método de enseñanza de español/L2 1 .

Por cuestiones históricas, la adquisición del español en pueblos indígenas es una imposición gubernamental por lo que sería necesario facilitar su aprendizaje. Del mismo modo, una competencia lingüística alta en la L2, podría aportar grandes beneficios a su integración en el grupo de la lengua meta. Debido a que, en muchas ocasiones, los comuneros se ven obligados a migrar a grandes ciudades en las que sufren una gran marginación por cuestiones lingüísticas, étnicas y culturales. A pesar de los "Propósitos de la Educación Básica Regular al 2021" recogidos en el Diseño Curricular Nacional de la Educación Básica Regular (2009) para facilitar la adquisición de la segunda lengua -protegiendo la L1- la enseñanza se lleva a cabo sin tener en cuenta la realidad lingüística del grupo objeto.

En este artículo se presentará una síntesis de los datos obtenidos en la investigación en cuanto al entorno en el que se desarrolla el proceso de enseñanza-aprendizaje, los factores contextuales que influyen en este y se ofrecerá material didáctico que ayude a facilitar la adquisición de la L2.

\section{Objetivos}

A pesar del multilingüismo existente en Perú, al español -el idioma oficial- se le confiere una mayor importancia en detrimento de las lenguas indígenas, por lo que no se favorece la adquisición de ambas lenguas y culturas al mismo nivel. Por ello, para mejorar esta situación se persiguen los siguientes objetivos.

-Estudiar y presentar la situación lingüística de las comunidades objeto.

\footnotetext{
${ }^{1}$ La investigación completa se puede consultar en: https://hera.ugr.es/tesisugr/26329694.pdf
} 
-Analizar el contexto en el que se desarrolla el proceso de enseñanza-aprendizaje de la L2.

-Diseñar alternativas didácticas realistas que se adapten al entorno del grupo objeto.

\section{Marco teórico-contextual}

\section{Situación lingüística}

En Perú no se produce una situación de equidad lingüística e, inevitablemente, este aspecto lleva a la marginación tanto de las lenguas minoritarias como de sus hablantes. El quechua no goza del mismo valor social que el idioma oficial, el castellano. Y, además, la variedad lingüística de la zona -español andino- es percibida por la sociedad hispanohablante de otras áreas del país de forma negativa.

La complejidad del escenario lingüístico que presenciamos nos lleva a la imposibilidad de elaborar un esquema conceptual concreto, debido a los numerosos elementos contextuales que influyen en la adquisición de la segunda lengua.

Si bien es cierto que se percibe un cierto patrón diglósico, producido por la convivencia de dos lenguas en desequilibrio en cuanto al valor social otorgado, en situaciones formales la L2 no es la lengua de comunicación constante. Sin embargo, es indiscutible destacar una cierta situación de bilingüismo como resultado del contacto entre ambas lenguas.

El bilingüismo no puede ser definido de forma concreta y unívoca ya que con el paso del tiempo han surgido numerosas acepciones según la perspectiva adoptada y la disciplina que lo estudia. Desde Bloomfield (1933), pasando por Weinreich (1953) hasta Blanco (1981), entre otros, nos encontramos con diferentes significados. En el presente artículo no nos detendremos a analizar de forma exhaustiva los innumerables estudios existentes en este campo, sino que sintetizaremos las perspectivas más relevantes en el caso que nos ocupa. De esta forma, resulta imprescindible destacar el bilingüismo aditivo y el sustractivo, acuñados por Lambert (citado en Postigo, 1998).

En el bilingüismo aditivo ambas lenguas son valoradas de igual forma y la adquisición de la L2 refuerza y valora el patrimonio lingüístico y cultural de la L1. El bilingüismo sustractivo, sin embargo, simboliza el objetivo lingüístico y cultural de una educación falsamente bilingüe (Postigo, 1998) y la adquisición de la L2 va en detrimento de la L1. Este tipo de bilingüismo ralentiza y dificulta el desarrollo de habilidades cognitivas y obstaculiza la adquisición de ambas lenguas.

De acuerdo a los datos recogidos en el INEI (Instituto Nacional de Estadística e Informática), aproximadamente el 55\% del departamento habla quechua, resultando el $45 \%$ hispanohablante. En las comunidades indígenas, exclusivamente, la media de quechua hablantes se incrementa y la de bilingües desciende notablemente. Aun teniendo en cuenta estas cifras, en el grupo objeto persisten carencias en las destrezas lingüísticas de ambas lenguas. Resulta evidente que para subsanar esta situación los programas de educación lingüística deberían adaptar el currículo a las características de la comunidad y tener en cuenta las diferencias de los grupos en contacto (contexto social, económico, cultural e histórico). Ya que, por el contrario, se estaría adoptando una enseñanza basada en el bilingüismo sustractivo y la comunidad perdería su lengua, cultura e identidad quechua (Zúñiga, 1993).

En los últimos años, teóricamente, se está aplicando un sistema de Educación Intercultural Bilingüe (EIB) en el país con el que se pretende adoptar una política lingüística y cultural que permita que los alumnos desarrollen competencias generales y lingüísticas, impartiendo el aprendizaje significativo en lengua vernácula y adquiriendo, al mismo tiempo, la L2. Lamentablemente, como iremos comprobando al analizar el material didáctico que se emplea en la enseñanza, la EIB no se cumple y no se produce una política lingüística real en las comunidades objeto. 
Por último, a esta compleja situación lingüística hay que añadirle un elemento fundamental que influye en la adquisición de la lengua, el analfabetismo; el cual a nivel de departamento está por encima del nacional y a nivel provincial llega a alcanzar el $50 \%$ en el caso de las mujeres y en más del $45 \%$ los habitantes que no concluyen los estudios primarios. En el grupo objeto, la tasa aumenta llegando al $53.3 \%{ }^{2}$ (mujeres>15 años).

\section{Factores que intervienen en la adquisición de la lengua en las comunidades objeto}

La adquisición de una segunda lengua está determinada por una serie de variantes comunes a todo contexto que influyen tanto en los procesos cognitivos como metacognitivos que intervienen en la misma. En el caso de las comunidades objeto, además, la situación social, económica y cultural determina absolutamente la competencia lingüística adquirida por los aprendientes. A continuación, se expondrán los puntos relevantes del entorno en el que se produce el proceso de enseñanzaaprendizaje.

A pesar de la importancia del patrimonio cultural e histórico del departamento y, por consiguiente, el amplio flujo de turistas que recibe, uno de los aspectos determinantes de las comunidades objeto es la situación económica en la que se encuentran. La alta tasa de pobreza lleva al departamento a estar entre los seis puestos con menor Índice de Desarrollo Humano del país (INEI, 2011). Este, entre otros, es uno de los motivos por los que la inclusión de nuevas tecnologías en el aula no resulta plausible.

Asimismo, es necesario poner de manifiesto la importancia que la alimentación tiene en el desarrollo integral de los niños y la repercusión de esta en la salud. La alimentación de los habitantes de las comunidades se basa primordialmente en el consumo de los productos que cultivan (papa, cebada, habas, maíz, chuño y khaya), por lo que no pueden mantener una alimentación equilibrada. Como consecuencia, se alcanza un alto índice de desnutrición crónica infantil (49\% en el distrito, superando la media regional y nacional. ${ }^{3}$ ) que repercute negativamente en las habilidades cognitivas y lingüísticas.

Prosiguiendo con el análisis de los elementos contextuales, es indispensable destacar otro aspecto fundamental: el trabajo infantil. En el "Plan Nacional de acción por la infancia y la adolescencia. Avances y retos del Ministerio de Trabajo y Promoción del Empleo (MTPE) (2002-2010)" se declara que el 28.6\% de niños (entre 6 y 17 años) trabaja. Resultando Cuzco el quinto departamento con mayor incidencia de trabajo infantil con un $50.7 \%$.

La mayoría de los niños que procede de zonas rurales trabaja para sus familias en tareas agrícolas y ganaderas pero, lamentablemente, también nos encontramos con otras formas de trabajo, el TID (Trabajo Infantil Doméstico). En esta categoría están incluidas las niñas que trabajan realizando tareas domésticas en casas ajenas al hogar familiar. La extenuante jornada de trabajo, el desarraigo familiar, la obligada desvinculación lingüística y cultural y los numerosos casos de abusos sexuales que sufren, someten a la población infantil a una terrible forma de explotación extendida en todo el país de forma latente (Organización Internacional del Trabajo, 2003).

Por último, existen varios elementos que repercuten en el ámbito educativo. Los medios con los que cuentan las escuelas son realmente escasos; por lo que los materiales en soporte digital no pueden llevarse al aula. Por otra parte, destacan las decadentes condiciones de las infraestructuras escolares: accesos inundados en temporada de lluvias; aulas con ventanas rotas y mobiliario en mal estado; suciedad en los servicios sanitarios; humedad concentrada en las aulas y una inadecuada

\footnotetext{
${ }^{2}$ Datos del Centro Yanapanakusun- Centro de Apoyo Integral a la Trabajadora del Hogar (CAITH). http://www.yanapanakusun.org/hg-caith/historia.htm

${ }^{3}$ Según estudios de Tdh Italia (Terre des Hommes) y el Centro de Apoyo Integral a la Trabajadora del Hogar (CAITH).
} 
temperatura, al prescindir de cualquier medio para calentar la clase. Por lo tanto, presentan una estructura que, además de ocasionar peligros para los escolares, afectan a su salud y no favorecen el aprendizaje. Por otro lado, debido a la ausencia de transporte, muchos niños se ven obligados a ir andando a la escuela realizando trayectos de entre 1-2 horas en caminos sin asfaltar.

En cuanto a la formación del profesorado, es fundamental señalar algunos datos relevantes. Desde 1964 nos encontramos con varias leyes que han determinado la Carrera Pública Magisterial (CPM). En la ley No 24029 -modificada-, en los artículos 64,66 y la quinta disposición transitoria queda reflejado cómo al principio los docentes no tenían que estar en posesión de un título profesional en educación y cómo podían enseñar con estudios de educación secundaria (Art.66 apartado e). A este dato hay que añadir que además, en muchas escuelas, hay un único docente que se encarga de cuatro grados - sin personal de apoyo- por lo que la calidad de la enseñanza se ve inevitablemente mermada.

A todo ello, en relación con el cuerpo docente, cabe destacar el fuerte absentismo laboral de los maestros que ejercen en medios rurales; la constante impuntualidad de los mismos y las frecuentes huelgas durante meses; acciones que influyen negativamente en el proceso de aprendizaje, viéndose interrumpido.

\section{Análisis de materiales didácticos y metodología}

Son muchos los métodos y los enfoques que se adoptan en la enseñanza de lenguas pero es difícil afirmar categóricamente cuál ofrece mejores resultados. Por ello, el objetivo -especialmente en contextos desfavorecidos- no es inclinarse hacia un modelo determinado, sino explotar cualquier tipo de recurso que pueda surtir efecto. Pese a ello, consideramos que el enfoque comunicativo fomentaría un uso real de la lengua y podría mejorar considerablemente las destrezas lingüísticas de los alumnos (Consejo de Europa, 2002). A este propósito cabe recordar que estamos ante un marco en el que la segunda lengua servirá como instrumento de comunicación también, y sobre todo, fuera del aula y en contextos no formales.

Por supuesto, al aplicar cualquier método, hay que adaptar el material para que se corresponda con los criterios del mismo. En este caso, para introducir un material que resulte comunicativo y se adapte a las necesidades del alumnado, es conveniente aplicar una serie de criterios de evaluación (Lozano y Ruiz Campillo, 1996). De estos, señalaremos aquellos que resultan fundamentales en la enseñanza en el grupo objeto. En primer lugar, resulta imprescindible que haya una relación entre la actividad y el mundo exterior, con el uso de materiales reales, tanto en la enseñanza de lenguas como de otras disciplinas. Es más, no solo tendríamos que apoyarnos en textos académicos propiamente dichos, sino que podríamos explotar incluso el entorno natural que nos rodea. Es decir, en nuestro grupo, sería más pragmático emplear la lengua en los mercados del distrito -con hispanohablantes- o aprender cálculos aritméticos en L2 en situaciones reales y no realizando divisiones imposibles con decimales, como sucede en las escuelas.

Otro criterio fundamental es la integración de las destrezas lingüísticas, sin necesidad de forzar su presencia en una misma tarea, resultando indispensable su introducción en el proceso de enseñanza global. Puesto que para alcanzar una cierta competencia lingüística hay que conseguir un equilibrio en todas las habilidades.

Asimismo, otro aspecto esencial es implicar al alumno, despertando su interés y motivándolo con actividades que respondan a sus características y necesidades y potencien la intervención comunicativa (Gardner, 2010). A este respecto, aun resultando evidente, cabe destacar que el grado de dificultad de las actividades debe corresponderse con el desarrollo intelectual, cognitivo y lingüístico de los alumnos.

Además de los aspectos destacados es fundamental tener en cuenta los factores contextuales, expuestos en apartados anteriores, para que el material responda a las necesidades del grupo y se adapte al mismo. 
A continuación, para concluir con el panorama general que rodea al ámbito educativo, describiremos sintéticamente la tipología de actividades realizadas en el aula y encontradas en manuales en uso en las escuelas ${ }^{4}$.

\section{Contenido}

La escuela podría ser un lugar idóneo para impulsar la adquisición de la L2, ya que acerca al niño a la lengua meta. Sin embargo, para ello, el material didáctico debe responder a las características del grupo. En primer lugar, resulta fundamental que el contenido de las actividades sea comprensible por parte del alumno y que se adecue a su desarrollo intelectual ya que, de lo contrario, el aprendizaje no se producirá. En los materiales analizados nos encontramos con contenidos totalmente desconocidos para niños quechua hablantes de zonas montañosas rurales. A menudo, en los manuales, aparecen lugares geográficos, entornos naturales -playas, mar- o contenidos herramientas informáticas- ajenos a su conocimiento del mundo. Asimismo, en estos textos destaca la complejidad léxica y gramatical que no se corresponde, en absoluto, con la competencia lingüística que un niño puede tener en la L2.

Es fundamental, por otra parte, que los temas elegidos sean de su interés para captar su atención y potenciar el interés por la L2; aspecto que no parece contemplarse en los textos analizamos.

Por último, destaca una marcada ausencia de cohesión temática y lingüística entre unidades de un mismo libro. Los contenidos se presentan de forma incongruente ya que se parte de un nivel inicial -deletreando palabras- y tras, tan solo, unas páginas se introducen textos de gran dificultad lingüística. Este hecho evidencia que los libros que se emplean en las escuelas no son de español/L2 sino para alumnos hispanohablantes.

\section{Metodología}

Las actividades del aula se basan principalmente en la repetición de palabras sin relación semántica entre sí -hasta 50-100 veces-, a modo de caligrafía. Se trata de tediosas copias de palabras cuyo criterio de selección parece basarse en grupos fonéticos o categorías gramaticales, aunque no siempre está claro. Este tipo de actividades no activa el uso de la lengua y solo se encarga de la parte ortográfica, aspecto que no favorece el desarrollo de destrezas lingüísticas en la L2.

Por otra parte, en cuanto a la corrección de las mismas, nos encontramos con tareas que han sido puntuadas de forma positiva, obviando los errores del alumno. Este hecho dificulta el aprendizaje por la desmotivación a la que están sometidos los niños y por la repetición de errores que los docentes no corrigen y que podrían llegar a convertirse en una fosilización. Asimismo, la desidia laboral de los maestros y las carencias en su competencia lingüística en español, las encontramos en fichas realizadas por ellos mismos en las que observamos numerosos errores ortográficos, gramaticales y léxicos con una escasa coherencia textual.

Una de las grandes dificultades a las que se enfrenta un profesor de lenguas es el error -cómo tratarlo o cuándo corregirlo- por ello, sería positivo trabajarlo junto a los estudiantes para que así pudieran formar parte activa en su aprendizaje y se potenciara al mismo tiempo la autonomía y el trabajo en grupo. Por otra parte, también podría elaborarse un registro con los errores más frecuentes, comunes, para presentarlos en clase (Giovanni et al., 1996) De esta forma, se podría afrontar la corrección sin desmotivar a los alumnos.

El dictado es otro de los recursos que los maestros suelen emplear en clase. Independientemente del actual debate sobre las ventajas o los inconvenientes del

\footnotetext{
${ }^{4}$ El análisis de la metodología y material didáctico se llevó a cabo durante la investigación realizada en el distrito de Huancarani, en las comunidades de Huancarani, Chinchayhuasi, Huaccaycancha y Huayllapata, desde octubre de 2012 a mayo de 2013.
} 
mismo, este método podría beneficiar a los alumnos promoviendo la atención, la memoria, identificando palabras y subsanando las interferencias fonéticas que se producen con su L1. Sin embargo, en los textos analizados, encontramos dictados con temas que no despiertan el interés de los alumnos y de una longitud excesiva -incluso de dos páginas- por lo que no se logra el objetivo didáctico.

En resumen, los materiales didácticos y la metodología empleada no favorecen la adquisición de la lengua. El alumno no es el protagonista de su propio aprendizaje y no tiene la posibilidad de interaccionar; además, se siente desmotivado y todo ello obstaculiza la posibilidad de alcanzar un cierto grado de bilingüismo.

\section{Propuesta didáctica fundamentada: Derecho a comunicar}

\section{TIC y enseñanza de lenguas: ¿cosas de ricos?}

Las nuevas tecnologías han repercutido en nuestra forma de relacionarnos y, de la misma manera, han provocado cambios en el modelo educativo al integrar herramientas digitales en el aula. El impacto de estas en sistemas educativos, ampliamente tradicionales, supuso un cambio en la enseñanza. Tanto por la falta de conocimiento sobre su aplicación, como por causas técnicas que impedían -estas últimas aún persisten- su puesta en práctica en el aula. Con el paso del tiempo, los efectos negativos se han ido paliando y actualmente contemplamos la rápida evolución de la tecnología y las ventajas de las TIC en la enseñanza de lenguas (Higueras, 2004) porque motivan, fomentan un trabajo colaborativo, favorecen diferentes tipos de aprendizaje y aumentan la participación de los alumnos.

En la historia de las Tecnologías de la Información y la Comunicación destacan, entre otros, la etapa de la tecnología analógica, la de la tecnología digital, la de la web social o web 2.0. Las redes sociales, relacionadas con esta última etapa, han alcanzado una gran importancia en el campo educativo y en la enseñanza de lenguas. Una red social es una estructura social -que se utiliza, generalmente, para comunicarse- formada por personas que tienen un vínculo en común: intereses, relación personal, familiar, profesional, etc. (Hernández, 2008). Esta forma de comunicación se puede llevar al terreno educativo, ofreciendo al alumno un papel activo en su aprendizaje y situándolo en el centro del proceso. Trabajar con redes sociales o con metodologías tan valoradas como las WebQuest (Adell, 2004) potencia un aprendizaje cooperativo entendido como un sistema de interacciones que organiza e induce la influencia recíproca entre los integrantes de un grupo (Johnson y Johnson, 1998), ya que los alumnos interaccionan entre sí para aportar materiales y compartir experiencias. En resumen, con el uso de una red social se crea una dinámica que potencia la motivación dentro y, especialmente, fuera del aula, favoreciendo un intercambio de opiniones. Además, el hecho de que los estudiantes tengan la oportunidad de compartir experiencias, comentar, publicar textos, fotos y noticias hace que se conviertan en protagonistas de su propio proceso de aprendizaje y desarrollen las destrezas lingüísticas y comunicativas.

Sin embargo, pese a la importancia de ofrecer recursos que resulten cercanos a nuestros alumnos, con un formato estético atractivo, no se pueden obviar los objetivos didácticos que se persiguen. La vorágine de recursos digitales, en la actualidad, a menudo ha supuesto un input desmesurado para el profesor de lenguas y esto, no en pocos casos, ocasiona una pérdida de dichos objetivos, obviando variables esenciales para que se produzca el aprendizaje. Es decir, para aprender una lengua, no basta con que nuestros alumnos creen un perfil en una red social, cuelguen fotos en Instagram, visualicen vídeos, empleen códigos QR, jueguen al Kahoot o realicen actividades tradicionales como rellenar huecos de frases incompletas, que en formato digital parecen didácticas, si no seguimos una programación. 
Del mismo modo, hay que examinar el entorno en el que se aplican las nuevas tecnologías, ya que de poco sirve llevar al aula material que nos obligue a hacer uso de estas en un lugar en el que apenas llega la luz eléctrica o con un grupo que desconoce la existencia de las mismas y el funcionamiento de dispositivos digitales. Por lo tanto, teniendo en cuenta que hay innumerables variables que influyen en la enseñanza, la metodología y el material necesariamente se tienen que adaptar.

\section{Derecho a comunicar: una propuesta didáctica humanista}

Tras los resultados obtenidos en la investigación surgió la necesidad de iniciar un proyecto que culminó en una propuesta didáctica cuyo objetivo principal radica en la mejora de la enseñanza de la L2. Previamente se han puesto de manifiesto los elementos contextuales que influyen en la adquisición de la lengua. Lamentablemente, los aspectos históricos, la desigualdad social, las dificultades económicas o la falta de desarrollo, no son fácilmente modificables; de ahí que la presente propuesta simplemente pretenda mostrar un método de enseñanza adaptado al medio en el que esta se lleva a cabo y al grupo al que va destinado.

Con el manual, a través de cinco capítulos, se trata de enseñar español mediante imágenes, juegos, trabajos manuales, etc. con un nivel que se corresponda con el desarrollo intelectual y lingüístico del grupo objeto. Se intentan cubrir las lagunas evidenciadas y potenciar habilidades generales de los niños, motivándolos e incluyéndolos en su aprendizaje.

A continuación, ejemplificaremos los objetivos didácticos principales que priman en el libro con la extracción de ejemplos del primer capítulo.

Las actividades elaboradas se corresponden con el desarrollo intelectual del niño y con el conocimiento que posee del mundo para insertar posteriormente otros conceptos y realidades. Se incluyen aspectos concernientes a la cultura indígena: nombres, pueblos o ciudades de su provincia y actividades propias de la zona. Con ello, además de presentar medios conocidos, se pone freno a la tendencia en la docencia actual que dirige hacia un bilingüismo sustractivo.

Al mismo tiempo, se fomenta la interacción oral, evitando las clases magistrales y la ausencia total de participación del estudiante. El maestro, en las comunidades, representa autoridad y poder $\mathrm{y}$, por este motivo, la posibilidad de intervención voluntaria de los niños es inexistente. Por consiguiente, predominan ejercicios en parejas, entrevistas o juegos de rol para ejercitar las habilidades comunicativas y fortalecer el trabajo en grupo. En cierto sentido, estas tareas se podrían equiparar a la introducción de redes sociales en la docencia en otros contextos en los que el entorno lo permite.

Hasta ahora se ha señalado la importancia de adaptar el material a la competencia lingüística del alumnado, entre otros. En este caso, además, es fundamental no perder de vista que los destinatarios son niños de educación infantil y primaria, respectivamente. Por esta razón, el uso de técnicas que atraiga su atención y fomente su creatividad cobra gran importancia. Emplear fotografías e imágenes, además de contribuir a almacenar información, ayuda a facilitar la comprensión y a aclarar conceptos culturales, lingüísticos o semánticos nuevos. Los dibujos que ilustran el manual han sido realizados exclusivamente en correspondencia con el objetivo de cada tarea, con la intención de que sirvan como medio para asociar mentalmente -de forma mnemotécnica- el vocabulario.

Se explota el dibujo de manera directa e inversa como herramienta didáctica. Es decir, se incluyen imágenes junto al término o concepto -a modo de pictograma- que sirva de aclaración y ayude a la deducción del mismo; o bien, se practica la comprensión lectora con textos, descripciones o poesías simples cuyo contenido nos lleva a realizar un dibujo, transformando el código lingüístico en imagen.

Al estudiar el entorno en el que se desarrollan los niños, se han destacado algunos elementos esenciales (alimentación, situación económica, trabajo, etc.). Asimismo, hay 
otros aspectos socioculturales que influyen en ellos. El ambiente no estimulante en el que crecen, hace que la psicomotricidad gruesa y fina se vean fuertemente retrasadas. La precariedad económica de las familias junto al concepto de educación y al modelo de conducta paternal lleva al abandono emocional de los hijos. Son principalmente las mujeres las que atienden al ganado y caminan en la montaña durante horas. Este hecho obliga a que trasladen a los niños - metidos en una manta- a la espalda sin poderles prestar la atención necesaria. De este modo, la evolución de la psicomotricidad se ralentiza y, en ocasiones, se refleja incluso en edad adulta no siendo posible subsanar estas carencias de la etapa infantil. Por consiguiente, manifiestan un retraso en el descubrimiento del cuerpo y en el desarrollo de movimientos primarios. Análogamente, la falta de afecto y cuidado, la ausencia de estímulos a los que están expuestos; la inexistente interacción con los otros y la carencia de expresión y recibimiento de emociones intervienen en la inteligencia emocional.

Para suplir estos aspectos, en el manual se tratan diferentes temas que pueden ayudar a los niños en este campo. Por una parte, en el primer capítulo, se trabaja específicamente el cuerpo humano con el objetivo de aportar actividades que permitan colaborar en la evolución de la psicomotricidad del niño desde el conocimiento del mismo. Por otra, se trata de ofrecer nociones básicas sobre el comportamiento y funcionamiento del cuerpo -a nivel físico y psicomotor- en L2 para ampliar su vocabulario. Resulta esencial que adquieran estos términos ya que los problemas de salud en la población son continuos. Asimismo, dado que la segunda lengua es el instrumento de comunicación con el grupo meta, es necesario ofrecer al grupo campos léxicos fundamentales y específicos para que se facilite la integración en este.

Durante la investigación los participantes mostraron grandes dificultades, en parte como consecuencia de la metodología adoptada en la enseñanza, a la hora de cumplimentar fichas básicas con datos personales, extraer información de textos o sintetizar ideas. Con el propósito de potenciar y trabajar habilidades generales -no solo destrezas lingüísticas- se han elaborado numerosas actividades con las que los niños aprendan a relacionar conceptos mediante conexiones visuales a través de la construcción de mapas conceptuales y esquemas. Se trata de que comprendan la información dada, sean capaces de sintetizarla y relacionen y agrupen conceptos por campos léxicos. Igualmente, se trabaja con series de palabras que favorezcan una asociación significativa, además de ampliar el léxico específico. En las copias que realizan en las escuelas, las listas de palabras se apoyan, generalmente, en grupos fonéticos sin conexión semántica impidiendo que se produzca un aprendizaje significativo. En general, los niños copian palabras de las que no conocen el significado e incluso se les dan palabras erróneas o inexistentes en español.

En resumen, este tipo de actividades no aporta elementos que favorezcan el desarrollo ni cognitivo ni lingüístico; arrastrando, obviamente, al alumno a un grado de desmotivación irreparable. Como consecuencia, el niño concibe el aprendizaje como un castigo y una imposición, sin disfrutar mínimamente de su etapa académica. El elemento lúdico en el aula es fundamental para evitar esta desmotivación y crear una atmósfera distendida que invite a la participación y facilite el aprendizaje. Desde una perspectiva histórica, son muchos los autores que han defendido el juego como herramienta educativa desde varias disciplinas, considerándolo fundamental en el proceso del desarrollo cognitivo y/o motivacional (Piaget, 1977; Vygotsky, 1997). Por ello, las actividades lúdicas están presentes en todo el manual, siendo uno de los recursos didácticos más significativos. El propósito de introducir el componente lúdico en el libro mediante actividades, tales como: jeroglíficos, trabajos manuales o sopas de letras; no radica solo en motivar al alumno en su aprendizaje, sino también en proteger otro de los derechos vulnerados del niño: el derecho a divertirse y a jugar. Debido a las numerosas obligaciones a las que están sometidos (trabajo, cuidado de sus hermanos), no disponen del tiempo necesario para poder cumplir con sus labores impuestas y disfrutar de su derecho infantil. Tras ocho meses de convivencia en 
dichas comunidades, se observó cómo los derechos del niño no son respetados ni en el núcleo familiar ni en el ámbito educativo. Por ello, en esta propuesta se han fortalecido los mismos y se ha tratado la educación en valores de forma interdisciplinar para educar a los niños en el respecto y la tolerancia e, indirectamente, hacer reflexionar a los adultos -profesionales en la educación- sobre la obligación ética de respetar los derechos fundamentales. A propósito de este último aspecto, cabe destacar que las actividades han sido diseñadas para que los alumnos puedan llevarlas a cabo sin la ayuda del docente, dado que para aplicar una cierta metodología tendría que producirse previamente un cambio en la perspectiva que los maestros de las comunidades poseen de la educación.

Por último, resulta relevante poner de manifiesto el componente interdisciplinar del libro, habiendo introducido conceptos propios de diversas áreas (ciencias sociales, ciencias naturales o lengua y literatura). En cuanto a la lengua en sí, no se ha adoptado un enfoque tradicional para explicar la gramática -este no era el objetivosino que, al tratarse de un contexto bilingüe se ha optado por la deducción de las estructuras y la futura asimilación de estas; independientemente de que se hayan tratado algunos aspectos relevantes. Asimismo, se trabajan temas esenciales -a través de la puesta en práctica de las destrezas lingüísticas integradas- relacionados directamente con la problemática del grupo: nutrición, alimentación, higiene, salud, etc. para que se adquieran conocimientos básicos fundamentales en su realidad.

\section{Discusión}

En el presente artículo se han extraído de manera sintética los factores relevantes que repercuten en el proceso de adquisición de español/ L2. Asimismo, se ha señalado cómo el ámbito educativo en el que se produce el proceso de enseñanza-aprendizaje, pone de manifiesto una serie de carencias que impide resultados efectivos. Posteriormente, se han resaltado sintéticamente los puntos relevantes relacionados con la metodología y los materiales didácticos que se emplean en las escuelas y las carencias del sistema. Y, por ello, para contribuir a mejorar la calidad educativa, se ha presentado Derecho a comunicar como propuesta didáctica.

En contextos desfavorecidos son muchos los factores contra los que no se puede luchar -pobreza, enfermedades, desigualdad social- pero sí podemos ofrecer una enseñanza de calidad, adaptando los materiales y el método de enseñanza para garantizar el éxito educativo. No podemos caer en la injusticia de pensar que todas las sociedades poseen los mismos recursos, ni que todas las culturas se rigen por las mismas pautas. Aunque, a priori, la ausencia de tecnología en el aula podría parecer una limitación en la enseñanza, en contextos en los que los recursos son mínimos, se puede lograr que se desarrolle el proceso de enseñanza-aprendizaje con materiales en diferentes soportes. Por supuesto, puede suponer un desafío, pero la educación es un derecho fundamental que no puede verse coartado o determinado por la desigualdad social. Por ello, ayudémosles a mantener el Derecho a comunicar.

\section{Referencias}

Adell, J. (2004). Internet en el aula: las WebQuest. Edutec, Revista Electrónica de Tecnología Educativa, 17, 1-26.

Blanco Abarca, A. (1981). Bilingüismo y cognición. Estudios de Psicología, 8, 50-81.

Bloomfield, L. (1933). Language. Nueva York: Holt. Trad. al español, Lenguaje. Lima: Universidad Mayor de San Marcos, 1964. 
Consejo de Europa (2002). Marco Común Europeo de Referencia para las Lenguas: aprendizaje, enseñanza, evaluación. Madrid: Instituto Cervantes-Ministerio de Educación, Cultura y Deporte-Anaya.

Fernández, S. (2001). Autonomía en el aprendizaje y enfoque por tareas. Frecuencia L, 17, 6-16.

Gardner, R.C. (2010). Motivation and second language acquisition: The socioeducational model. Nueva York: Peter Lang Publishing.

Giovannini, A., Martín Peris, E., Rodríguez, M. y Simón, T. (1996). Profesor en acción 2. Madrid: Edelsa.

Hernández Requena, S. (2008). El modelo constructivista con las nuevas tecnologías: aplicado en el proceso de aprendizaje. RUSC. Revista de Universidad y Sociedad del Conocimiento. Vol. 5, 2, 26-35.

Higueras, M. (2004). Internet en la enseñanza del español. En Vademécum para la formación de profesores. Enseñar español como segunda lengua (L2)/Lengua extranjera (LE) (1061-1085). Madrid: SGEL.

Johnson, D. W. y Johnson, R. (1998). Cap. 2: Cooperative learning, values, and culturally plural classrooms. En M. Leicester, C. Modgill, y S. Modgil (Eds.), Values, the classroom and cultural diversity (15-28). Londres: Cassell PLC.

Lozano, G. y Ruiz Campillo, J.P. (1996). Criterios para el diseño y la evaluación de materiales comunicativos. Didáctica del español como lengua extranjera. Cuadernos del tiempo libre. Colección Expolingua. ELE 3. Fundación Actilibre, 127-155.

Ministerio de Educación (2009). Diseño Curricular Nacional de la Educación Básica Regular. Lima (Perú).

Piaget, J. (1977). La formación del símbolo en el niño. México: Fondo de Cultura económica.

Postigo, R. M. (1998). Modelos de educación lingüística en contextos de lenguas en contacto. En A. Mendoza (coord.), Conceptos clave en didáctica de la lengua y la literatura (265-274). Barcelona: Horsori.

Vygotsky, L. S. (1997). El papel del juego en el desarrollo del niño. Revista de educación física: Renovar la teoría y práctica, 66, 5-11.

Weinreich, U. (1953). Lenguas en contacto. Descubrimientos y problemas. Caracas: Universidad Central de Venezuela, 1974.

Zúñiga, M. (1993). Educación Bilingüe: Materiales de apoyo para la formación docente. En Educación Intercultural Bilingüe. La Paz, Bolivia: UNICEF. 